\title{
Morphological variations of human adult spleens in a cohort of Sri Lankan population: A cadaveric study
}

\author{
Abarana $\mathrm{K}^{1}$, Samaranayake UMJE ${ }^{1}$, Anthony $\mathrm{DJ}^{1}$ \\ ${ }^{\prime}$ Department of Anatomy, Faculty of Medicine, University of Colombo
}

\begin{abstract}
Introduction

Spleen is an organ enriched in vascular and lymphoid tissue located in left hypochondrium. It has morphological variations which may be misinterpreted as a disease condition involving the spleen. Therefore, knowledge on such morphological differences in a population is vital to distinguish normal variations from the disease conditions. Objective of this study was to identify the morphological variations such as notches, fissures and lobulations in cadavers.
\end{abstract}

\section{Methods}

Ten-percent formalin fixed, selfdonated cadaveric human spleens $(n=13)$ in the Department of Anatomy, Faculty of Medicine, University of Colombo were included in the present study. In the study population, male to female ratio was 10: 3 with age ranging from 26 to 95 years. The morphological features such as shape, notching of borders, fissures and lobulations were photographed, dissected and analyzed according to Michels NA classification. The morphological types of spleens were categorized by measuring hilar lengths.

\section{Results}

Notches were present in superior $(\mathrm{n}=8)$, inferior $(n=1)$ or intermediate $(n=2)$ borders in ten spleens, while notches were absent in three. Five spleens had supernumerary notches. Six spleens contained fissures in both visceral and diaphragmatic surfaces. Four spleens had more than three lobules, while three had more than one hilum. The splenic types included compact, intermediate and distributed, which accounted for four, three and six respectively.

\section{Conclusion}

Inconstant location of notches, presence of supernumerary notches, fissures and lobules were a common morphological variation observed.

Keywords: cadavers, morphology, spleen

\section{Introduction}

Spleen is an intraperitoneal, encapsulated organ enriched with vascular and lymphoid tissue. It usually found in the left hypochondrium under the left dome of the diaphragm. The extent of the spleen is from the ninth to eleventh rib. 
Abarana K, Samaranayake UMJE, Anthony DJ - Morphological variations of human adult spleens in a cohort of Sri Lankan population: A cadaveric study

The embryonic development of the human spleen is not fully understood. Around the $5^{\text {th }}$ week of gestation, in the dorsal mesogastrium the mesenchymal cells proliferate to form a mesenchymal mass which gives rise to hematopoietic tissues. By the 8th week, the spleen has a segmented morphology of lobules, which gradually disappear around week 30 as the spleen develops its lymphoid structures. A few lobules eventually fuse to form the spleen proper.

During development, due to its relationship with the surrounding structures, the shape of spleen varies from crescentic to quadrangular [1]. It has a visceral and a diaphragmatic surface. Visceral surface is located at the undersurface of the stomach, with impressions for stomach, colon, left kidney and pancreas. Its diaphragmatic surface is completely covered by peritoneum and usually does not contain fissures [1]. The visceral surface is also covered by peritoneum, except at the hilum which allows blood vessels to enter and exit.

It has three margins; superior, inferior and intermediate. The superior margin of the spleen possesses characteristic notches. The notches located in the superior border of the spleen are representatives of growth that separated the lobules during fetal life [2]. Notches however, can be observed in inferior and intermediate margins as well [1]. The position and curvature of the hila are variable.

Michels NA classification categorizes the spleens according to its morphology as compacted, intermediate and distributed by measuring the length of the hilum in centimeters [3].

Spleen has morphological variations in different populations, which may be misinterpreted as various disease conditions involving the spleen. Therefore, knowledge on such morphological differences in a population is vital to distinguish normal variations from the disease conditions. Objective of this study was to identify the morphological variations such as notches, fissures and lobulations in cadavers.

\section{Material and methods}

This descriptive cross-sectional pilot study using human cadavers was conducted at the Department of Anatomy, Faculty of Medicine, University of Colombo, Sri Lanka from April 1, 2019 to May 1, 2019. This study included 13 cadavers that were $10 \%$ formalin fixed, self-donated, with a male: female ratio of 10:3 of and with age ranging from 26-95 years. Human spleens that had destructed surfaces and margins by any mechanical, pathological or other conditions were excluded from the study.

The spleens were inspected macroscopically in detail for shapes, surfaces, borders, hilum and presence or absence of accessory spleen and lobulation. Each spleen sample was photographed perpendicular to the specimen using a 16.0 mega pixel 
Abarana K, Samaranayake UMJE, Anthony DJ - Morphological variations of human adult spleens in a cohort of Sri Lankan population: A cadaveric study

camera. (Pictures available in Figure 1)

The presence of notches on the superior, inferior and intermediate borders and the presence of fissures on the diaphragmatic or visceral surface were noted. Spleens were analyzed according to Michels NA classification. The morphological types of spleens were categorized by measuring hilar lengths.

Standard descriptive statistics were used to analyze the data using SPSS software.

\section{Results}

Among the 13 spleens (Figure 1) 4 were oval, 3 triangular, 3 wedge, 1 tetrahedral, 1 elliptical and 1 crescent spleen were noted. Notches were present in superior $(\mathrm{n}=8)$, inferior $(\mathrm{n}=$ 1) or intermediate $(n=2)$ borders in ten spleens, while notches were absent in three. Five spleens had supernumerary notches. Six spleens contained fissures in both visceral and diaphragmatic surfaces. Four spleens had more than three lobules, while three had more than one hilum. The splenic types included compact $(\mathrm{n}=4)$, intermediate $(\mathrm{n}=6)$ and distributed $(\mathrm{n}=3)$, which accounted for four, three and six respectively.

\section{Discussion}

Different populations are found to have numerous morphological variations of the spleen. Notches in the superior border was a common finding in many studies (Table 1) which was a similar finding in ours.

The average number of superior border notches to be reported is 2-4 [5]. In our study we observed two spleens, one with four and another with three notches in the superior border. However, in rare instances it is possible to have superior notches up to nine as reported by Thanya et al [4].

In a Malaysian study, deep fissures extending to the diaphragmatic surface were sparse and it occurred only in $1 \%$ of cases [5]. In an Ethiopian Study of 21 spleens, in two specimens $(9.52 \%)$, the deep notches were observed on the superior and inferior borders extended towards the diaphragmatic surface in the form of two fissures [6]. In our study, six spleens had fissures extending to diaphragmatic surface. Out of them, three had two fissures. Nayak et al [8] reported a lobulated spleen with five fissures and this is the highest number of fissures reported in the literature.

Out of 32 spleens of a North Indian population, 3 spleens $(9.3 \%)$ had multiple lobules [9]. In the present study, 3 spleens had five lobules, 1 spleen had four lobules, 3 had three lobules, 3 had two lobules and 2 had single lobule.

Ivan described, Lobulations of the spleen as a normal variation of spleen shape and it was not associated with any pathological conditions. However, these lobulations may be misinterpreted as splenic lacerations in patients with abdominal trauma [10]. Also, splenic 
Abarana K, Samaranayake UMJE, Anthony DJ - Morphological variations of human adult spleens in a cohort of Sri Lankan population: A cadaveric study

lobulations may be misdiagnosed as gastric or left renal tumors [11]. This is further proven by Smidt [12] with the presence of a congenital fissure mimicking splenic hematoma.

Abnormal lobulations, therefore could be misinterpreted as a mass originating from the kidney by the radiologists [11]. Therefore, we believe that the findings of our study are of great importance with regards to treatment during trauma care. Table 2 compares on incidence morphologic types of spleen based on Michels NA classification [13] with other studies. In our study more than one hilum could be seen in three spleens. A study in Kerala, also mentions multiple hila. Similarly, there were other studies that reported multiple hila [13].

Presence of supernumery notches, fissures and lobules were a common anatomical variation in this cohort of cadavers of Sri Lankan origin. Studies done in future with a larger sample size will aid in the identification of common morphological variations in the Sri Lankan population.

\section{Acknowledgements}

The authors thank the deceased and his family and the anatomy department laboratory staff members for their support in conducting the research.

\section{Corresponding Author}

Dr. Abarana Kiritharan

Department of Anatomy, Faculty of

Medicine, University of Colombo, Sri

Lanka.

Email address:

abarana2001@gmail.com

Phone number: +94712093534

\section{References}

1. Standring, S., Borley, N. R., \& Gray, H. (2008). Gray's anatomy: the anatomical basis of clinical practice. 40th ed., anniversary ed. [Edinburgh]: Churchill Livingstone/Elsevier.

2. Langman's Medical Embryology 11th ed., Sadler, T W, (Thomas W.); Langman, Jan. Philadelphia: Wolters Kluwer Lippincott Williams \& Wilkins, c2010.

3. Michels NA. The variational anatomy of the spleen and splenic artery. American Journal of Anatomy. 1942 Jan; 70(1):21-72.

4. Pathirana, Thanya \& Barton, Matthew \& George, Mark \& Forwood, Mark \& Palagama, Sujeewa. (2016). A rare anomaly of the human spleen with nine notches associated with multiple accessory spleens. A case study, hypothesis on origin and review of clinical significance. Italian journal of anatomy and embryology = Archivio italiano di anatomia ed embriologia. 121. 
Abarana K, Samaranayake UMJE, Anthony DJ - Morphological variations of human adult spleens in a cohort of Sri Lankan population: A cadaveric study

5. Das, Srijit \& Latiff, Abdul \& Haji Suhaimi, Farihah \& Ghazalli, Hairi \& Othman, Faizah. (2008).

Anomalous splenic notches: A cadaveric study with clinical importance. Bratislavské lekárske listy. 109. 513-6.

6. Tenaw B, Muche A. Assessment of anatomical variation of spleen in an adult human cadaver and its clinical implication: Ethiopian cadaveric study. Int J Anat Var. Dec 2018;11(4):139-142.

7. Patil GV, Shishirkumar, Apoorva D, Thejeswari, Sharif J, C Sheshgiri et al. Study of Splenic notches in a human cadaver. International Journal of Recent Advances in Multidisciplinary Research. 2014; 1(2):

8. NAYAK, B. S.; SOMAYAJI, S. N. \& SOUMYA, K. V. A Study on the variations of size, shape and external features of the spleen in South Indian population. Int. J. Morphol., 29(3):675-677, 2011.

9. D. M. H. D. K. H. Dr. Birendra Yadav, Dr. Nema Usman, Anatomical variations of spleen in North Indian population and its clinical significance, IJMHS, vol. 3, no. 4, Oct. 2013.
10. Varga, Ivan \& Babala, Jozef \& Kachlík, David. (2017). Anatomic variations of the spleen: current state of terminology, classification, and embryological background. Surgical and radiologic anatomy: SRA. 40.

11. Yildiz, Adalet \& Ariyurek, Macit \& Karcaaltincaba, Musturay. (2013). Splenic Anomalies of Shape, Size, and Location: Pictorial Essay. The scientific world journals. 2013. 321810 .

12. Paul Smidt K. Splenic scintigraphy: a large congenital fissure mimicking splenic hematoma. Radiology. 1977 Jan;122(1):169.

13. Nayak, S. B., Shetty, P., R, D., Sirasanagandla, S. R., \& Shetty, S. D. (2014). A lobulated spleen with multiple fissures and hila. Journal of clinical and diagnostic research: JCDR, 8(9), AD01-AD2. doi:10.7860/JCDR/2014/8996.4774 